\title{
Research and Integration of IoT based Solar Photovoltaic Panel Health Monitoring System
}

S. S. S. Ranjit, Senior Lecturer, Centre for Telecommunication Research \& Innovation, Fakulti Kejuruteraan Elektronik dan Kejuruteraan Komputer (FKEKK), Universiti Teknikal Malaysia Melaka (UTeM), Hang Tuah Jaya, 76100 Durian Tunggal, Melaka, Malaysia.

M. Abbod, Reader, Department of Electronic and Computer Engineering, College of Engineering, Design and Physical Sciences, Brunel University London, Kingston Lane, Uxbridge, Middlesex UB8 9PH, United Kingdom.

\begin{abstract}
This paper describes about the research and integration of IoT based solar photovoltaic panel health monitoring system. The proposed system comprises of four thermocouple K-Type sensors each one is connected to adafruit MAX31855 thermocouple amplifier, one INA219 adafruit voltage/current sensor and Raspberry Pi Zero Wireless. The thermocouple sensors each one is connected to adafruit MAX31855 thermocouple amplifier are installed at the back on the solar photovoltaic panel at measured distance between the sensors to sense the solar photovoltaic panel's temperature. The one INA219 adafruit voltage/current sensor is connected at the positive output wire of solar photovoltaic panel to sense and measure the amount of voltage/current produced. The sensed and measured values at the thermocouple sensors and voltage/current sensor are recorded into the Raspberry Pi Zero Wireless temporary storage. The recorded data in the Raspberry Pi Zero Wireless is then pushed into cloud system to ease the solar photovoltaic panel health monitoring and analysis via the website. This paper will explain the overall research and integration output of the proposed project and validate the recorded results based on the developed methodology.
\end{abstract}

Keywords--- PV Monitoring, Preventive Maintenance, Remote Monitoring, Real-Time Monitoring. 


\section{Introduction}

Electricity is the most basic requirement for everyone in today's modern world [1]. Due to the requirement the electricity energy consumption have shown large utilization and increment [2]. Hence, concurrently the fossil-fuel sources used to generate the electricity is diminishing as well [3]. Also, the volatility of oil prices as well as increasing worldwide concern on the carbon dioxide $\left(\mathrm{CO}_{2}\right)$ emissions has led towards the evolution of renewable energy such as solar, wind, hydro etc concepts based systems over the recent years [4]. At different part of the world and based on the climate nature, respected renewable energy source type of system has been developed to accommodate their increasing energy requirement. One of the most emerged and appropriate renewable energy system is solar photovoltaic (PV). The PV system has been continuously gaining attention among the players in the solar industry all around the globe [4], [5]. With the growing demand among the players in solar industry, the manufacturing and deployment solar photovoltaic have increase drastically in this recent years [4].

As solar photovoltaic system emerged as most promising technology to meet the demand for electricity growth [6], monitoring PV system is necessary to allow their holders to maintain and observe the installed PV system [7]. Another reason to monitor the PV system is because solar PV systems are installed at location where is not accessible to grid network electricity and installed solar PV system at such locations tend to be installed with monitoring system [8]. Such locations also have lack of technical manpower to perform operation check and maintenance on the solar PV system. This also hinder one to regularly check the system performances and record the output data [8]. Looking at the briefly discussed restrictions, it is necessary to install PV system with monitoring system to allow the system to perform up to the mark [8]. Also monitoring system would also assist one into carrying out the preventive maintenance when it is necessary.

In [8] is mentioned that due to lack of schedule service and maintenance, PV system tend to fail and the failure can be subjected to many related components. One of the frequently failed component is photovoltaic panel performances. Prieto in [4], proposed wireless sensor network based system for individual monitoring of panels. This system sensed meteorological and solar radiation data to optimize energy production based on surrounding ambient temperature and condition. In another research [6], monitoring PV system is developed. This system comprises of acquisition layer, pretreatment and recording layer and supervision, storage and web services layer. The acquisition layer consists of wireless sensor network that collect the information from the solar panel and meteorological. Next layer is pretreatment and recording, which manages the receive data from the wireless sensor network and temporary store the received data into the data logger. Then the temporary stored data into the data logger is then send to supervision, storage and web service layer [6]. In [9] microchip PIC18F4550 microcontroller is used to develop the proposed system. The system proposes to allow the users to check the photovoltaic panel temperature and radiation which satisfies the performance of the photovoltaic panel based on the datasheet. Research by M. Vyas [10], uses Labview software to monitor the photovoltaic panel's cell output voltage and at the same time provides updates on the system's status. In another research [11], a system integrated with pyrometer and temperature sensor is introduced. This system monitors the solar irradiance and temperature of the system, as well as is used to perform further analysis on the developed system. In [12], data acquisition system which records real-time data such as voltages from solar panel and track the system using sunlight information using the Arduino on the LabVIEW Makers Hub software.

In this context, the main objective of this paper is to research and integrate the IoT scheme to monitor the photovoltaic panel health condition which can assist to gather the real-time photovoltaic panel information. The research is to understand the current available technology for solar photovoltaic system and in some specific finding in regards to photovoltaic panels too. Understanding this research finding helps this research to develop the new methodology for it's proposed idea.

The remaining of the paper has been arranged as following: 1) Overview of the Proposed System, 2) Methodology of Hardware Development and Integration, 3) Results and Discussions, 4) Conclusion and 5) Acknowledgement.

\section{Overview of the Proposed System}

As explained, due to the increasing electricity demand and requirement to monitor the performances of the solar photovoltaic system, each component of the system has to be monitored. Hence, this paper proposes an IoT based Solar Photovoltaic Panel health monitoring system. This section will explain and describe the conceptual development of the proposed idea.

Figure 1 shows the conceptual development idea and suggestion of sensors placement at the back of the photovoltaic panel. Four units of thermocouple sensors with adafruit max31855 thermocouple amplifier and one INA219 adafruit voltage/current sensor are integrated with the Raspberry Pi Zero Wireless. The conceptual design is developed based on the study carried out in the introduction section. The thermocouple sensors with adafruit 
max31855 thermocouple amplifier is placed at the back of the photovoltaic panel to sense and measure the generated heat on photovoltaic panel top. The voltage/current sensor is connected in series at the positive wire of the photovoltaic panel to sense the produced voltage and current. All of these sensors are then connected to the Raspberry Pi Zero Wireless.

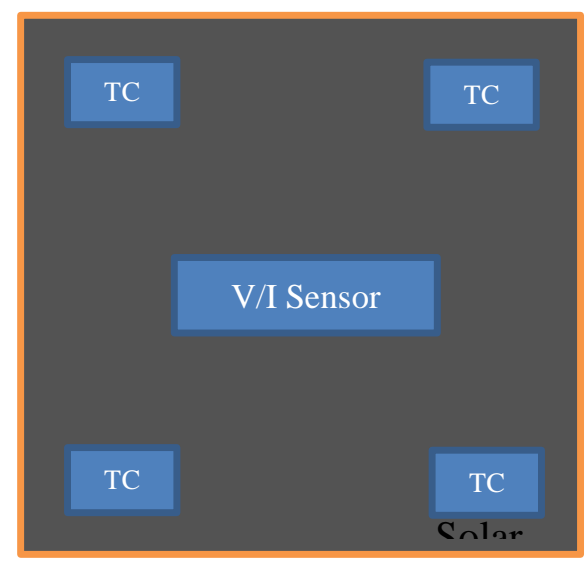

Photovoltaic Panel

- Tcp: Thermocouple Sensors

- V/I: Voltage/Current Sensor

Figure 1: Top View of Conceptual Development.

Once the conceptual development has been finalized and all the sensors and system connectivity have been decided, the conceptual idea needs to be illustrated for it to be seen and understandable. Hence, Figure 2 shows the conceptual development idea discussed in Figure 1 is illustrated using the fritzing software. All the sensors connection are also strictly followed as explained earlier to avoid any mistake. Solar panel and battery is used to show the complete system connectivity and the sensors arrangement according to the system objective.

Table 1 is explaining the connectivity referencing for thermocouple sensors with adafruit max31855 thermocouple amplifier and one INA219 adafruit voltage/current sensor with the Raspberry Pi Zero Wireless. The "THERMO1, THERMO2, THERMO3 and THERMO4" thermocouple sensors are attached at the back of the photovoltaic panel. While the input of the INA219 voltage/current sensor is connected at the positive wire of the photovoltaic panel.

Table 1: Connectivity Thermocouple Sensors

\begin{tabular}{|l|l|}
\hline Wiring Colour & \multicolumn{1}{|c|}{ Description } \\
\hline & VCC - Power Supply 3.3V, 5V, 12V \\
\hline & GND - Ground \\
\hline & SCL - Serial Data \\
\hline & SDA - Serial Clock \\
\hline & DO - Digital Output \\
\hline & CS - Chip Select \\
\hline & CLK - Clock \\
\hline
\end{tabular}

Reference for

\section{Connection of INA219 Adafruit Voltage/Current Sensor with Raspberry Pi Zero Wireless}

- $\quad$ Connect Pi 3.3V to INA219 VCC

- Connect Pi GND to INA219 GND

- Connect Pi GPIO 02 to INA219 SDA

- Connect Pi GPIO 03 to INA219 SCL 


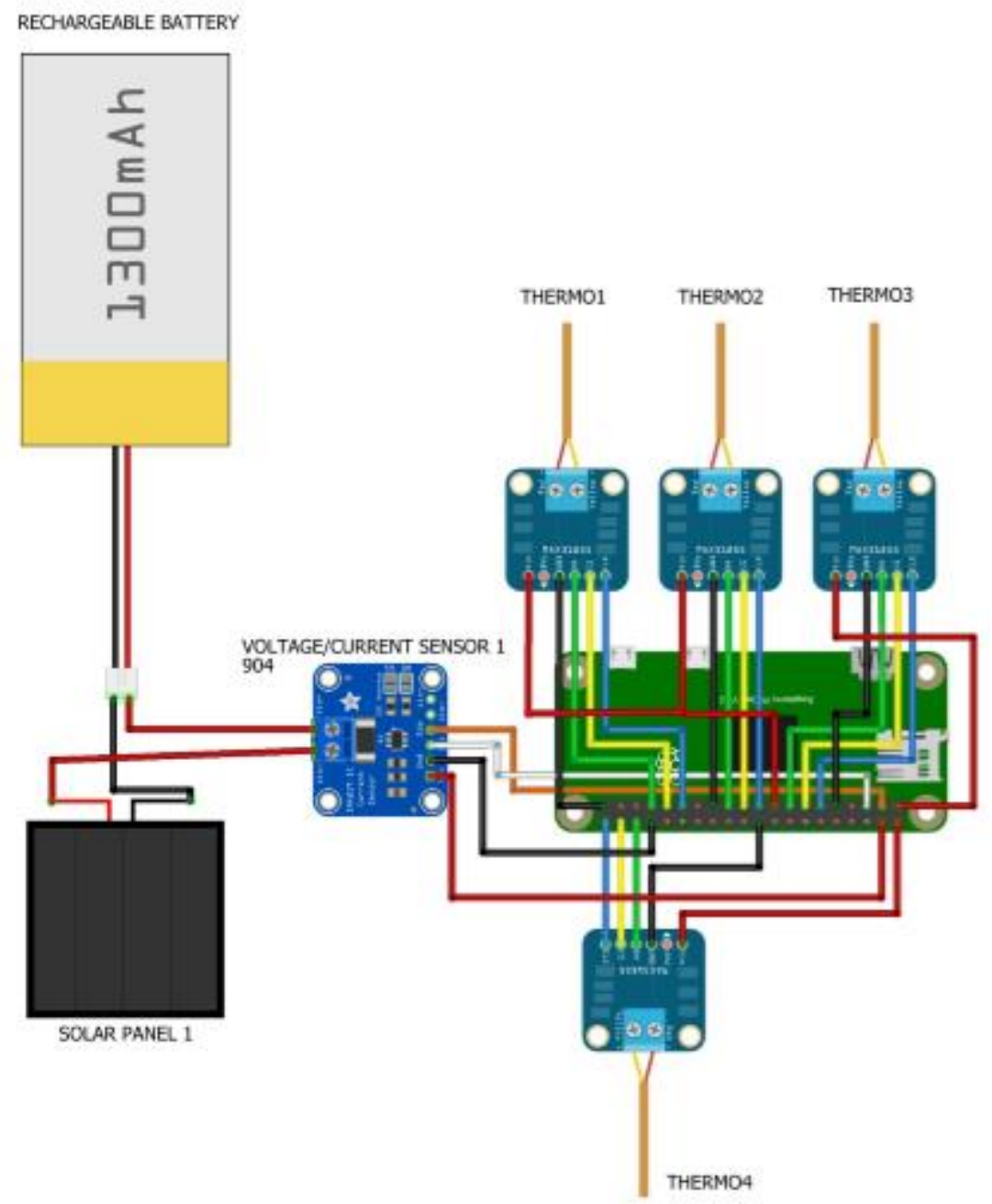

Figure 2: Sensors Integration and System Architecture Design using Fritzing Software.

\section{Connection of Thermocouple K Sensors with Adafruit MAX31855 Thermocouple Amplifier with Raspberry Pi Zero Wireless}

Connection of Adafruit MAX31855 thermocouple Amplifier

- Connect Raspberry Pi Zero Wireless Pin 3.3V to MAX31855 Vin

- Connect Raspberry Pi Zero Wireless Pin GND to MAX31855 GND

Connection Thermocouple Amplifier (TC1):

- Connect Raspberry Pi Zero Wireless Pin GPIO 22 to MAX31855 DO

- Connect Raspberry Pi Zero Wireless Pin GPIO 27 to MAX31855 CS

- Connect Raspberry Pi Zero Wireless Pin GPIO 17 to MAX31855 CLK

Connection Thermocouple Amplifier (TC2):

- Connect Raspberry Pi Zero Wireless Pin GPIO 11 to MAX31855 DO

- Connect Raspberry Pi Zero Wireless Pin GPIO 09 to MAX31855 CS

- Connect Raspberry Pi Zero Wireless Pin GPIO 10 to MAX31855 CLK

Connection Thermocouple Amplifier (TC3): 
- Connect Raspberry Pi Zero Wireless Pin GPIO 23 to MAX31855 DO

- Connect Raspberry Pi Zero Wireless Pin GPIO 24 to MAX31855 CS

- Connect Raspberry Pi Zero Wireless Pin GPIO 18 to MAX31855 CLK

Connection Thermocouple Amplifier (TC4):

- Connect Raspberry Pi Zero Wireless Pin GPIO 07 to MAX31855 DO

- Connect Raspberry Pi Zero Wireless Pin GPIO 12 to MAX31855 CS

- Connect Raspberry Pi Zero Wireless Pin GPIO 08 to MAX31855 CLK

\section{Methodology of Hardware Development and Integration}

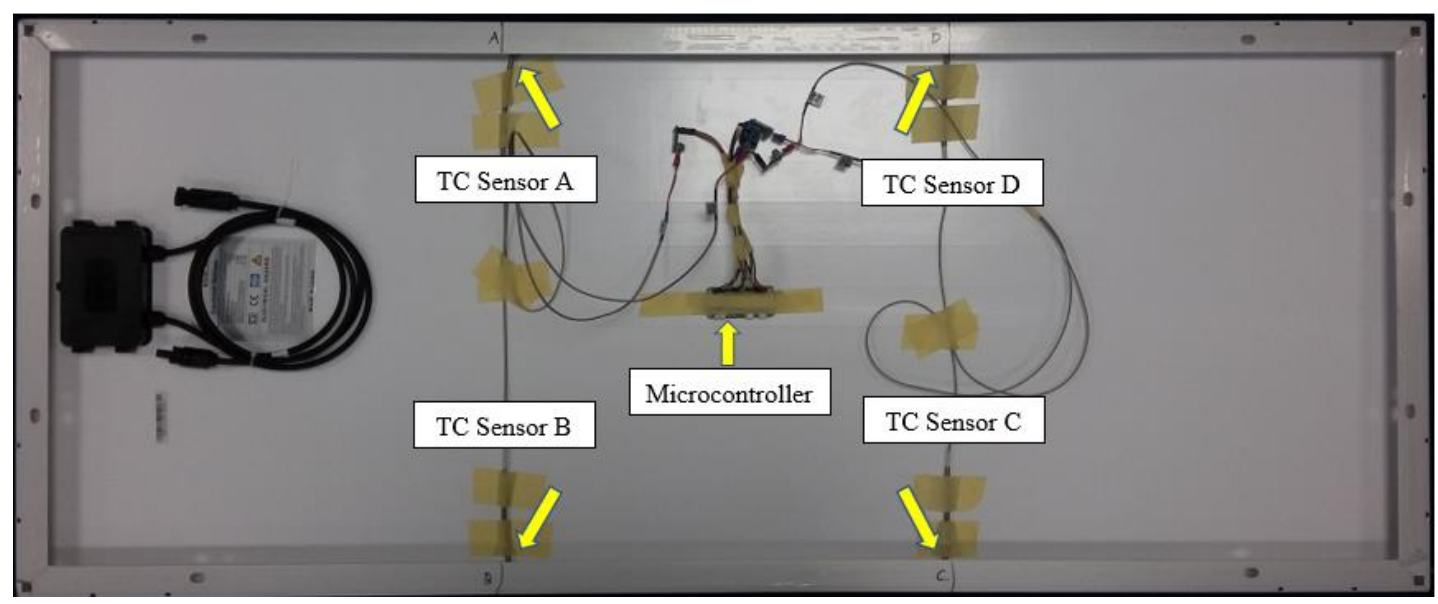

Figure 3: Placement and Integration of Thermocouple Sensors at the back of Photovoltaic Panel.

This section explain and show the methodological integration process of the proposed IoT based solar photovoltaic panel health monitoring system. The thermocouple K-Type sensors (TC Sensor A, TC Sensor B, TC Sensor C and TC Sensor D) with adafruit MAX31855 thermocouple amplifier is attached at the back of the photovoltaic panel. These sensors are connected to the adafruit MAX31855 thermocouple amplifiers before connecting to the Raspberry Pi Zero Wireless. The adafruit MAX31855 thermocouple amplifier is used to amplify the small signal received from the thermocouple $\mathrm{k}$ type and store the readings into the temporary storage in the Raspberry Pi Zero Wireless. The INA219 adafruit voltage/current sensor is used to sense and measure the output voltage at the respective photovoltaic panel.

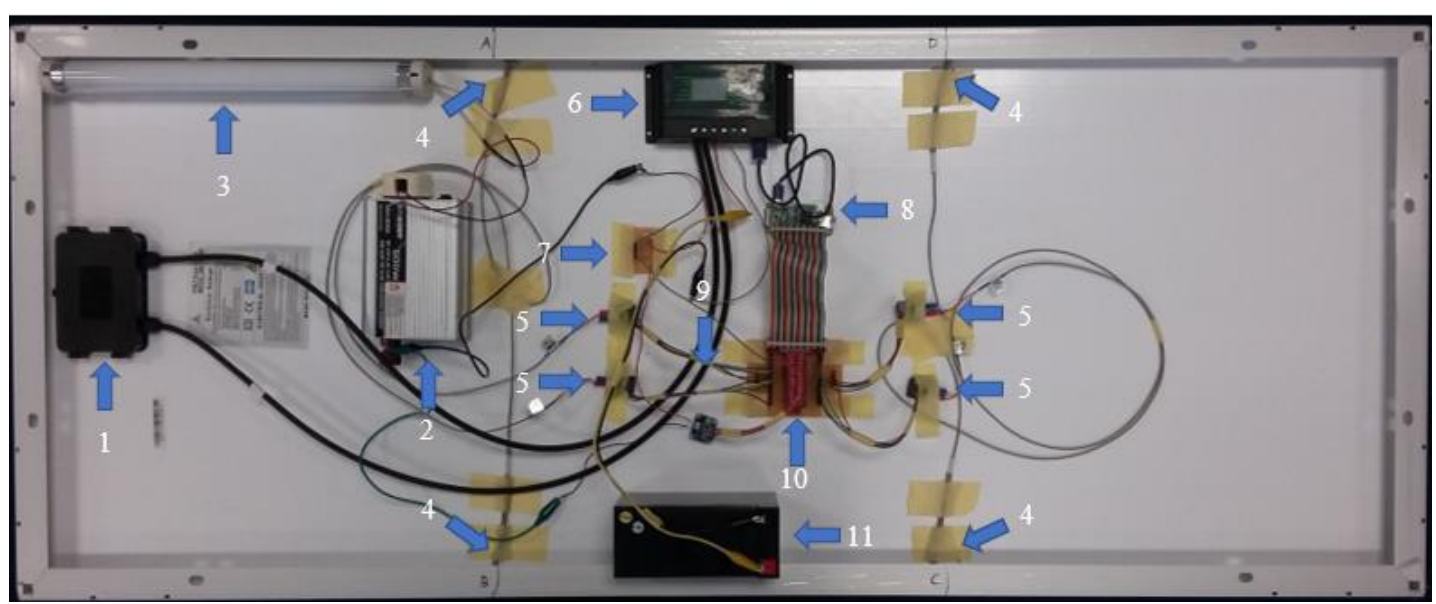

Figure 4: Complete Hardware System Integration and Installation 
Table 2: Reference Table for Figure 4

\begin{tabular}{|c|l|}
\hline Number Component & \multicolumn{1}{|c|}{ Hardware } \\
\hline $\mathbf{1}$ & Solar Panel \\
\hline $\mathbf{2}$ & Solar Power Inverter DC 12V to AC 220V \\
\hline $\mathbf{3}$ & Lamp \\
\hline $\mathbf{4}$ & Thermocouple K-Type (A, B, C, D) \\
\hline $\mathbf{5}$ & Thermocouple Amplifier (MAX31855) \\
\hline $\mathbf{6}$ & Solar Charger \\
\hline $\mathbf{7}$ & PCB Current Divider \\
\hline $\mathbf{8}$ & Raspberry Pi Zero Wireless \\
\hline $\mathbf{9}$ & Voltage/Current Sensor (INA219) \\
\hline $\mathbf{1 0}$ & PCB GPIO 40 Pins \\
\hline $\mathbf{1 1}$ & Rechargeable Battery 12V \\
\hline
\end{tabular}

In the following section the results recorded for the thermocouple K-Type sensor integrated with adafruit MAX31855 thermocouple amplifier and INA219 adafruit voltage/current sensor is presented. The results shows that the thermocouple K-Type sensor and INA219 voltage/current sensor has effective managed to achieve the objectives to record the photovoltaic panel data to observe the photovoltaic panel's health.

\section{Results and Discussions}

\section{a. Thermocouple K-Type Sensor and INA219 Voltage/Current Sensor}

The presents the sensed and measured results for the thermocouple K-Type sensor and INA219 voltage/current sensor. The presented reading in Figure 5 shows reading taken before $11 \mathrm{a} . \mathrm{m}$. and after $11 \mathrm{a} . \mathrm{m}$. The reading taken after 11 a.m. is recorded till 6p.m. in the evening. Based on the Figure 5 reading shows the temperature of the photovoltaic panel increases due to environment temperature increase.

Figure 6 presents the sensed, measured and calculated power at the photovoltaic panel. According to the trend, the voltage and current before $11 \mathrm{a} . \mathrm{m}$. stays constant, starts decreasing after 11a.m. When the voltage and current pf the photovoltaic panel decreased after 11a.m., the output power also decreases.

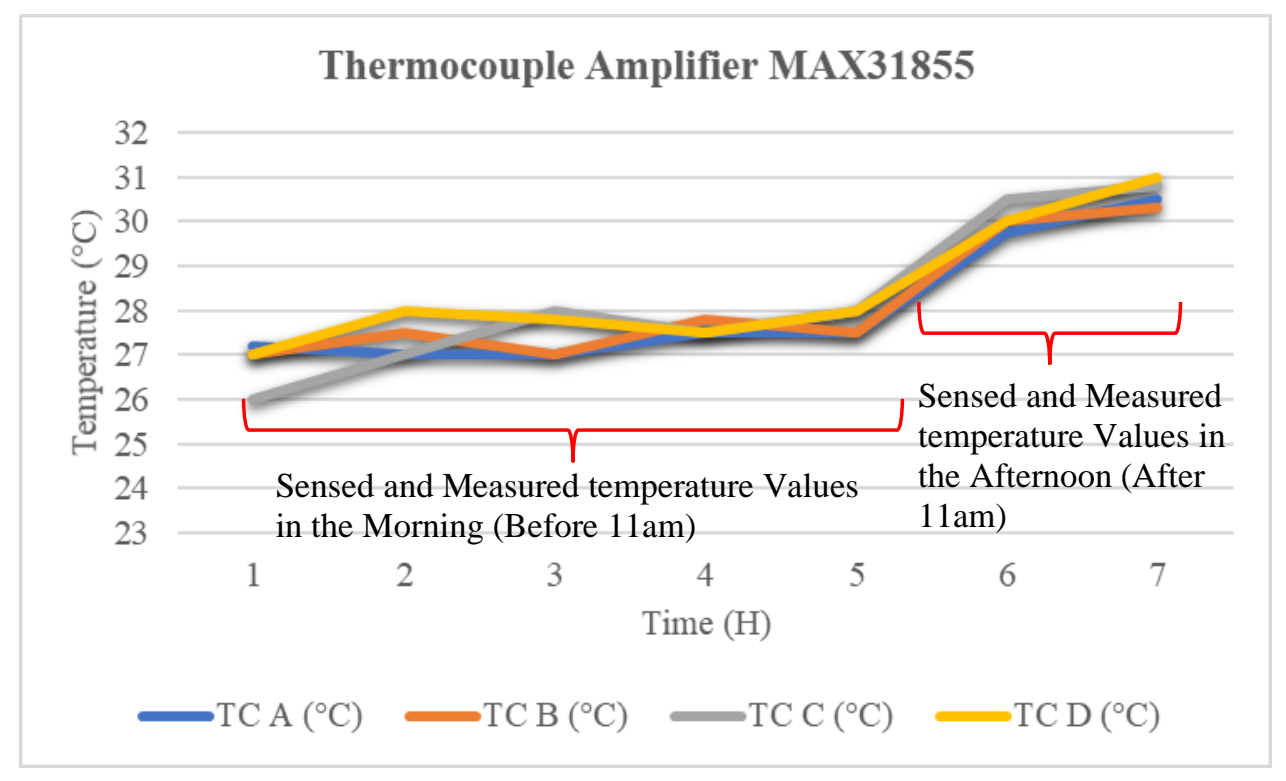

Figure 5: Sensed and Measured Temperature at Photovoltaic Panel. 


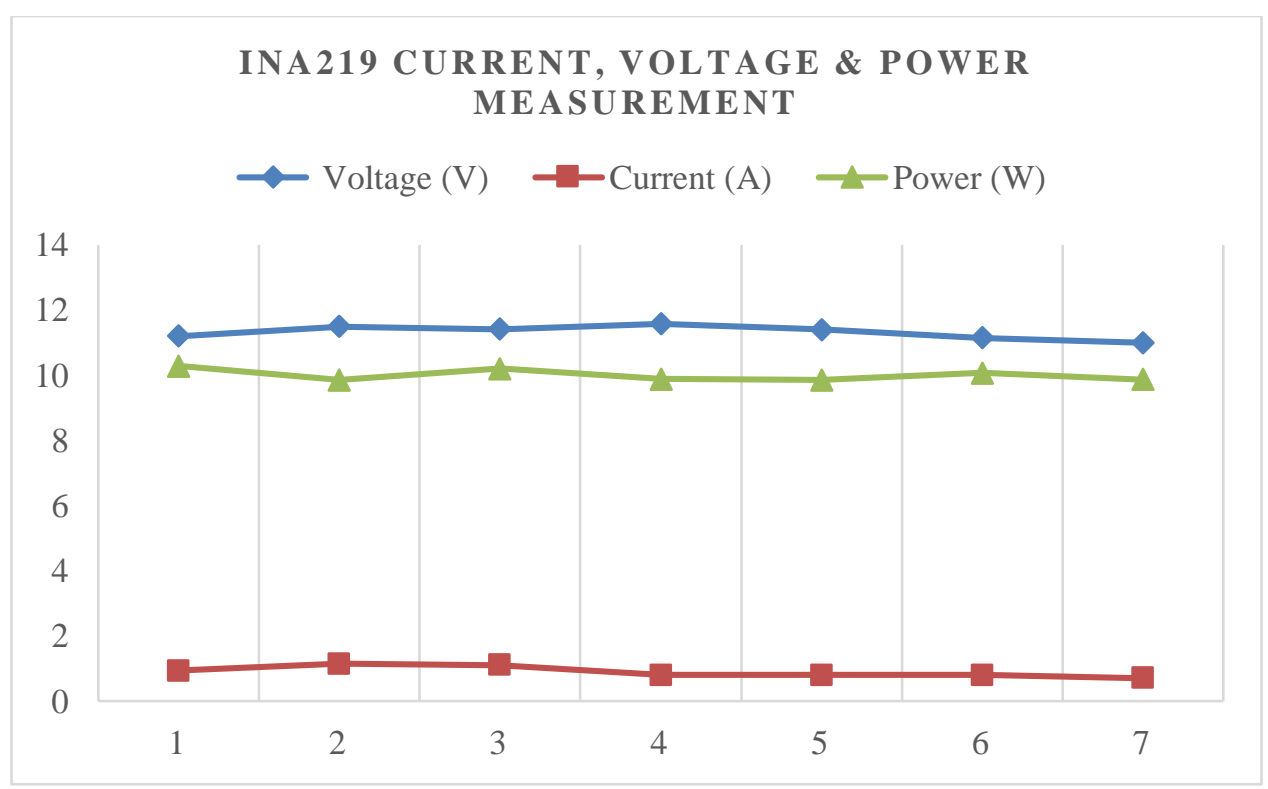

Figure 6: Sensed and Measured Voltage, Current and Calculated Power.

\section{b. Google Cloud - Current, Voltage and Temperature}

This section presents the recorded results of voltage, current and temperature at the google cloud which is setup using the google drive. Figures 7 and 8 shows the voltage, current and temperature values for INA219 voltage/current sensor and all the FOUR (4) thermocouple k-type sensors are recorded into the google cloud. These data is initially recorded in the SD Card at the Raspberry Pi Zero Wireless before being pushed into the google cloud.

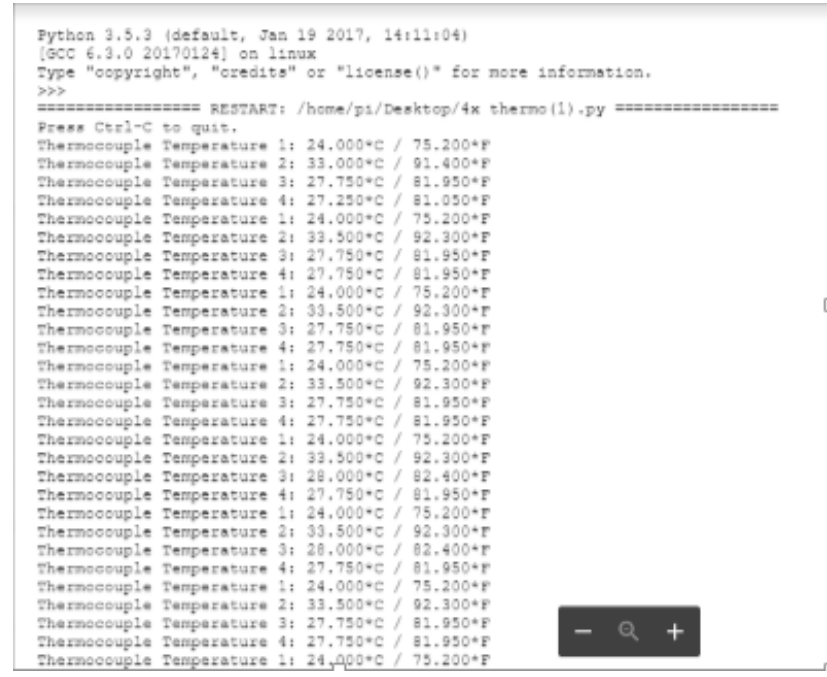

Figure 8: Photovoltaic Panel Temperature Recorded using Thermocouple K-Type Sensor at Google Cloud.

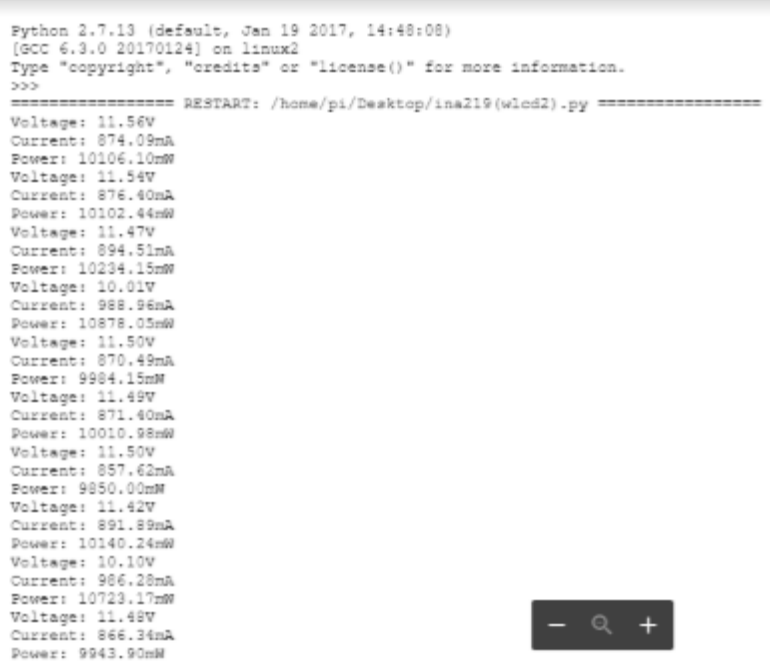

Figure 7: Recorded Voltage, Current and Calculated Power Recorded at Google Cloud.

\section{Conclusion}

This paper presents the conceptual and methodological process of setting up IoT based solar photovoltaic panel health monitoring system for an individual photovoltaic panel. From measured and recorded results, the real-time voltage, current and temperature recorded into the SD Card of Raspberry Pi Zero Wireless is sent to the google cloud for consumers viewing. The presented voltage and current results are varying with respect to the environment condition which also indicate the respective photovoltaic panel's health condition. The results recorded into the google cloud also shows that the recorded data at the SD Card of Raspberry Pi Zero Wireless is successfully transfer via the 
wireless network technology. Apart from that, the successfulness of implementing the IoT based solar photovoltaic panel health monitoring system shows that the technological implementation could help to reduce the fatigue at the photovoltaic panels and could be easily detectable when any fatigued condition occurs at photovoltaic panel.

\section{Acknowledgement}

This research is carried out under the research grant No: PJP/2017/FKEKK-CETRI/S01559. The author(s) wish to thank the Ministry of Higher Education of Malaysia (MOHE), Centre for Telecommunication Research \& Innovation (CeTRI), Fakulti Kejuruteraan Elektronik dan Kejuruteraan Komputer (FKEKK), Universiti Teknikal Malaysia Melaka (UTeM), Melaka, Malaysia and Brunel University London, United Kingdom.

\section{References}

[1] R. Singh, M. Abbod, and W. Balachandran, "Low Voltage Hybrid Renewable Energy System Management for Energy Storages Charging- Discharging," IEEE Explor., no. Dc, pp. 6-11, 2016.

[2] R. Tejwani, G. Kumar, and C. Solanki, "Remote Monitoring for Solar Photovoltaic Systems in Rural Application using GSM Voice Channel," Energy Procedia, vol. 57, no. 1959, pp. 1526-1535, 2014.

[3] P. Sindhuja and M. S. Balamurugan, "Smart Power Monitoring and Control System Through Internet of Things using Cloud Data Storage," Indian J. Sci. Technol., vol. 8, no. 19, 2015.

[4] M. J. Prieto, A. M. Pernía, F. Nuño, J. Díaz, and P. J. Villegas, "Development of a Wireless Sensor Network for Individual Monitoring of Panels in a Photovoltaic Plant," Sensors (Switzerland), vol. 14, no. 2, pp. 23792396, 2014.

[5] R. S. S. Singh, M. Abbod, and W. Balachandran, "Simulation of a New Proposed Voltage-Base SelfIntervention Technique with Increment and Decrement Voltage Conduction Method to Optimize the Rrenewable Energy Sources DC Output," Proc. - 2016 UKSim-AMSS 18th Int. Conf. Comput. Model. Simulation, UKSim 2016, pp. 224-229, 2016.

[6] S. Manzano, D. Guevara, and A. Rios, "An Overview of Remote Monitoring Pv Systems : Acquisition , Storages , Processing and Publication of Real- Time Data Based on Cloud Computing," 4th Sol. Integr. Work., no. November 2014, 2016.

[7] L. Saraiva, A. Alcaso, P. Vieira, C. F. Ramos, and A. M. Cardoso, "Development of a Cloud-Based System for Remote Monitoring of a PVT Panel,” Open Eng., vol. 6, no. 1, pp. 291-297, 2016.

[8] R. Tejwani, G. Kumar, and C. S. Solanki, "Remote Monitoring of Solar PV System for Rural Aare as using GSM, V-F \& F-V Converters," J. Instrum., vol. 11, no. 5, 2016.

[9] S. Sousa et al., "Implementation of a Low Cost Data Acquisition Board for Photovoltaic Arrays Analysis and Diagnostic," no. October, pp. 20-23, 2013.

[10] M. Vyas, K. Chudasama, M. Bhatt, and B. Gohil, "Real Time Data Monitoring of PV Solar Cell using LabVIEW," Int. J. Curr. Eng. Int. J. Curr. Eng. Technol., vol. 6, no. 6, pp. 2218-2221, 2016.

[11] S. Parthasarathy and N. V. Anandkumar, "Development of Low Cost Data Acquisition System for Photo Voltaic System," Int. J. Innov. Res. Sci. Eng. Technol., vol. 5, no. 7, pp. 12850-12856, 2016.

[12] S. Mandal and D. Singh, "Real Time Data Acquisation of Solar Panel Using Arduino and Further Recording Voltage of the Solar Panel," Int. J. Instrum. Control Syst., vol. 7, no. 3, pp. 15-25, 2017. 